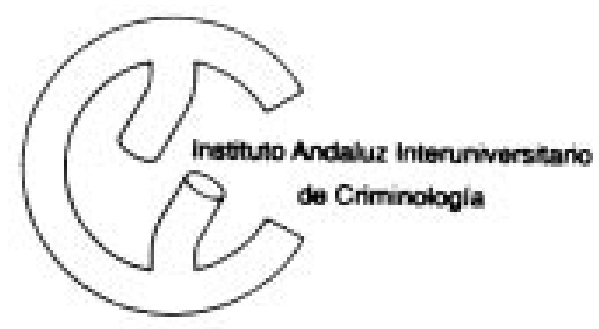

Director: Per Stangeland

Coordinadora: $\mathrm{M}^{\mathrm{a}}$ José Garrido de los Santos

Publicado por la Sección de Málaga del IAIC

Facultad de Derecho, Universidad de Málaga

Campus de Teatinos, 29071 MALAGA

Tel:(95) 2132325 - Fax:(95) 2132242

Depósitolegal:MA857/1996ISSN:1137-2427

En el Boletín Criminológico no 34 tuve ocasión de describir las particularidades jurídicas, sanitarias y experimentales que presentaba el primer proyecto español de administración controlada de heroína a heroinómanos con un determinado historial de fracaso en otros tratamientos. La iniciativa de la Consejería de Asuntos Sociales de la Junta de Andalucía sigue aparentemente tropezando con una actitud poco comprensiva de los órganos de la Administración central competentes para autorizar el Programa. En estos momentos se está a la espera de que la Dirección general de Farmacia del Ministerio de Sanidad reconsidere su decisión de no autorizar el experimento, una vez que el organismo impulsor de su realización lo ha corregido de forma que ya no presente el par de defectos técnicos que fundamentaron la denegación de la autorización.

Para entretener la espera, este Boletín presenta los esperanzadores resultados de un estudio sobre los efectos que el pionero proyecto suizo de administración controlada de heroína ha tenido sobre la delincuencia de las personas sometidas al programa experimental. El informe completo de este estudio se publicará en un próximo número de la Revista de Derecho Penal y Criminología.

José Luis Díez Ripollés

Director del I.A.I.C.

\title{
La prescripcion de heroína como medida de prevención de la delincuencia: Resultados de la experiencia suiza
}

El programa suizo de prescripción de heroína

Las políticas de reducción de riesgos consideran que los toxicómanos bien integrados en la sociedad tienen más posibilidades de abandonar la droga que aquellos que viven marginalizados y, en consecuencia, recomiendan la aplicación de medidas de asistencia médico-social que permitan facilitar la reinserción social de las personas en fase de dependencia. Como ejemplo de dichas medidas podemos citar la distribución de jeringuillas estériles, la creación de centros de ayuda en materia de alojamiento, alimentación y trabajo, así como los programas de dispensación de metadona y de opiáceos.

En Suiza, un programa piloto de prescripción de heroína fue autorizado por el Consejo Federal (órgano colegiado que detenta el poder ejecutivo) en 1992. Este programa está destinado a los heroi- nómanos más intensamente implicados en el consumo y para quienes las terapias convencionales han fracasado. Mediante el suministro de heroína, acompañado de una asistencia médica, psicológica y social, se intenta mejorar el estado de salud de las personas tratadas y eliminar el estado de tensión provocado por la necesidad de procurarse cotidianamente (y en muchos casos por medios ilegales) el dinero necesario para adquirir la droga.

El programa, para el cual ochocientas plazas fueron habilitadas y ocupadas en su totalidad, recibió la aprobación de una comisión de ética y comenzó en enero de 1994. A partir de julio de 1996 no se aceptaron nuevos pacientes, pero se continúa tratando a las personas inscritas. Las condiciones de admisión exigen una edad mínima de 20 años, un historial de adicción a la heroína superior a dos años, al

\section{Autores: \\ Marcelo F. Aebi \\ Martín Killias}

menos dos intentos fallidos de abandonar dicha adicción con otros métodos, y la presencia de daños a nivel físico o social debidos al consumo de drogas.

El tratamiento es ambulatorio y se lleva a cabo en 18 pequeños centros de prescripción que permiten un contacto directo con el paciente y facilitan las medidas de ayuda médico-social, complemento indispensable del tratamiento. Luego de haber tomado contacto con el paciente, un médico establece la dosis diaria que le será suministrada. Dicha dosis debe ser consumida en el centro de prescripción. Además, cada participante paga diariamente un monto de 15 francos suizos (1 franco suizo $=100$ pesetas) como contribución al tratamiento.

Este programa fue objeto de numerosas evaluaciones sobre sus aspectos médicos, económicos y sociales. Se han estudiado los

\section{Boletín \\ Criminológico \\ $N^{o} 39$ Febrero \\ 1999 \\ Página


efectos de las sustancias utilizadas, así como los efectos del tratamiento sobre la salud, la situación socioeconómica de los participantes y la delincuencia ligada al consumo de drogas. La evaluación de este último aspecto fue confiada al Institut de police scientifique et de criminologie (IPSC) de la Universidad de Lausanne.

En este contexto se estudió la evolución de la prevalencia (es decir del porcentaje de personas que cometen delitos) y la incidencia (el promedio de delitos cometidos por cada individuo) de la delincuencia mediante una comparación de los delitos cometidos por las personas tratadas antes y después de su admisión en el programa. A tal fin se utilizaron como indicadores de la delincuencia las condenas penales, los contactos con las fuerzas policiales y unas encuestas de delincuencia autorrevelada y de victimización. La combinación de estos indicadores permite obtener una imagen mucho más completa de la delincuencia que aquella que se obtendría con sólo alguno de ellos puesto que es sabido que todos los indicadores de la delincuencia presentan diversos inconvenientes que les impiden reflejar de manera exacta el fenómeno criminal.

Antes de comenzar a describir la metodología y los resultados de la evaluación realizada, señalemos que la base teórica de las políticas de reducción de riesgos está emparentada con las teorías criminológicas que destacan el rol de las oportunidades en la explicación del delito. Básicamente, estas teorías se inspiran en el refrán que sostiene que "la ocasión hace al ladrón". En consecuencia, no se proponen cambiar al hombre (como lo hacen la mayoría de las teorías criminológicas) sino reducir las ocasiones que se le presentan para cometer delitos. En el caso que nos ocupa, se parte de la hipótesis que los toxicómanos que reciben la heroína no van a recurrir a la delincuencia para procurarse el dinero necesario para comprarla.
Condenas penales y contactos con las fuerzas policiales

Los colaboradores del IPSC consultaron el Registro Federal de condenas y recabaron todas las informaciones pertinentes sobre los participantes en el programa. De esta manera fueron codificadas las condenas de las que fueron objeto, con indicación de la fecha en que fueron pronunciadas, de los delitos incluidos y de las sentencias aplicadas.

Con respecto a los contactos con las fuerzas policiales, es necesario señalar que Suiza no cuenta con un registro central de policía, sino que cada cuerpo de policía cantonal (y en algunos casos municipal) mantiene su propio registro. De este modo pueden existir varios legajos para un mismo delincuente. En consecuencia, para poder acceder a la información sobre los participantes en el programa, los colaboradores del IPSC se desplazaron personalmente a los diez cuerpos de policía de los cantones y ciudades que participaron en el programa y accedieron en cada uno de ellos al registro establecido a nivel individual en el que son indicados los contactos que cada persona ha tenido con la policía en calidad de sospechoso de un delito. Este control puso en evidencia que la mayoría de los participantes en el programa eran conocidos de las fuerzas policiales y en ciertos casos sus legajos eran extremadamente vo- luminosos (con un promedio de más de 6 contactos anuales con la policía para el grupo presentado en la figura 1). Se codificó todo contacto registrado desde la admisión en el programa, así como todo aquel registrado en los dos años inmediatamente anteriores a ésta, con indicación de la fecha y del tipo de infracción. También fue indicado el número total de contactos durante los cinco años anteriores al último período indicado.

Tanto en el caso de las condenas penales como en el de los contactos con las fuerzas policiales, la confidencialidad de los datos personales de los participantes fue respetada al máximo. A cada uno de ellos se le asignó un número interno y los datos fueron consignados en fichas individuales, identificadas por dicho número.

Como puede observarse en la figura 1, a partir de la entrada en el programa se produce una disminución muy marcada de los contactos con la policía, así como de las condenas penales y de los delitos objeto de tales condenas. En el caso de los contactos con la policía la disminución se sitúa en el orden del $60 \%$, mientras que en el caso de las condenas penales ésta es del orden del $80 \%$. Señalemos además que la duración de las penas de prisión impuestas se redujo en un $33 \%$.

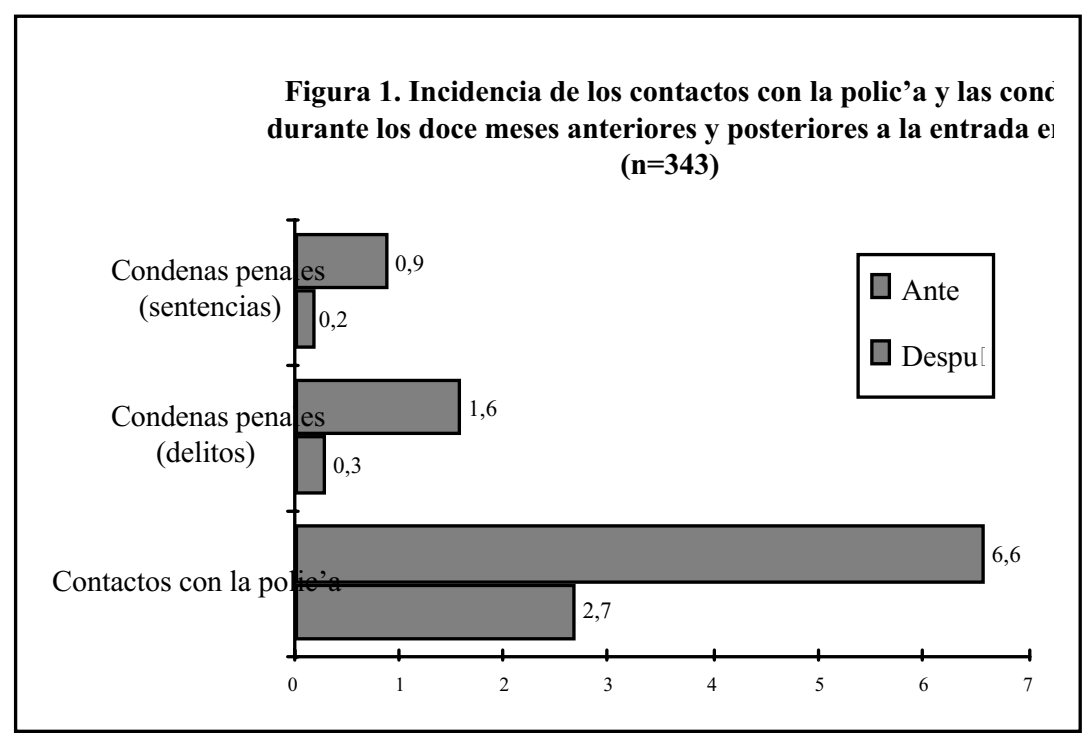


Figura 2. Prevalencia de la delincuencia autorrevelada antes de la entrada en el progl despu $\square$ s de un a-o en tratamiento (per'odo de referencia: los 6 meses anteriores a entrevista correspondiente; $n=319$ )

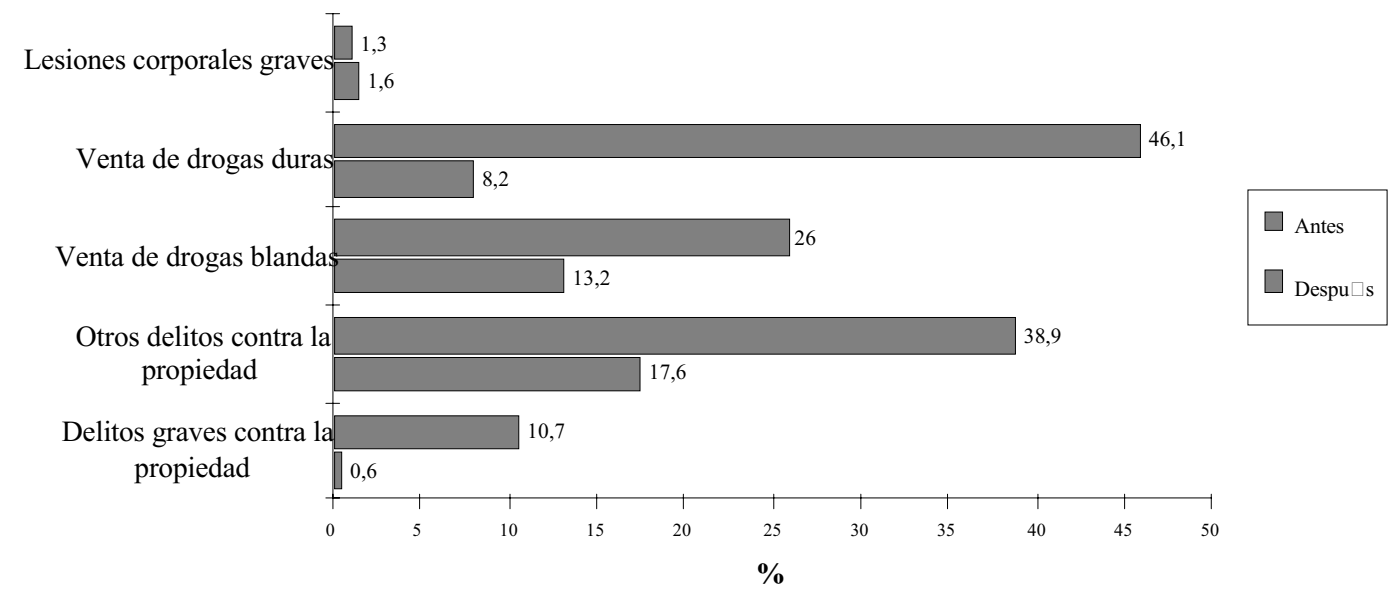

\section{Encuestas de delincuencia au- torrevelada}

Las encuestas de delincuencia autorrevelada permiten obtener informaciones pormenorizadas sobre cada infracción y las circunstancias que la rodean, detalles que escapan regularmente a los registros policiales y judiciales.

Las entrevistas se realizaban por primera vez en el momento de la entrada en el programa y a intervalos de seis meses a partir de esa fecha. La persona encuestada recibía una lista de 12 preguntas (que describían la comisión de otros tantos delitos) a responder por escrito de manera afirmativa o negativa. Luego informaba del código de las respuestas afirmativas al encuestador (que no conocía el tipo de delito sino únicamente su código), quien le hacía las preguntas de detalle. Estas preguntas permitían recoger datos relevantes sobre el delito cometido, por ejemplo, la frecuencia de comisión en los últimos seis meses y las últimas cuatro semanas, la edad a la cual se delinquió por primera vez, las características de la víctima (en el caso en que la hubiera), el método aplicado y los contactos con la policía como consecuencia del delito.

La figura 2 presenta la evolución de la prevalencia de los delitos contra la propiedad (los delitos "graves" incluyen todo tipo de robos con violencia o intimidación, fuerza en las cosas y carterismo; mientras que los robos de menor gravedad y la venta de cosas robadas han sido reagrupados en la categoría "otros"), la venta de drogas blandas y duras, y las lesiones corporales graves con y sin armas.

Los resultados obtenidos muestran una disminución significativa de la prevalencia de la delincuencia. Esta reducción es particularmente importante en el caso de los delitos graves contra la propiedad y el tráfico de drogas duras, mientras que es menos marcada en el caso de los robos de menor gravedad. El escaso número de delitos de lesiones corporales (los resultados se basan en los datos de 5 personas como máximo) confirman que se trata de un delito poco común entre los toxicómanos y excluye la posibilidad de obtener resultados significativos a nivel estadístico.

La disminución de las tasas de incidencia es aún más importante, con reducciones de aproximadamente el $90 \%$ para todo tipo de delitos contra la propiedad y para el tráfico de drogas duras. Esto significa que aquellos toxicómanos que continuaron delinquiendo redujeron la cantidad de delitos cometidos.

Encuestas de victimización e

\section{influencia del estilo de vida}

Las entrevistas que hemos detallado en el apartado precedente comenzaban con una encuesta de victimización. Esta encuesta fue incluida porque numerosas investigaciones han mostrado que el riesgo de ser víctima de un delito depende en gran parte del estilo de vida de una persona. De esta manera se ha comprobado que los delincuentes son víctimas de delitos con mayor frecuencia que el resto de la población. Este fenómeno se explica por el ambiente marginal que suelen frecuentar, donde abundan otros delincuentes.

La encuesta de victimización puede entonces ser utilizada como medio indirecto para evaluar la delincuencia de los participantes. Por este motivo fueron incluidos en ella algunos delitos estrechamente ligados al estilo de vida de los toxicómanos, acompañados de otros que son en principio independientes de dicho estilo. También se incluyeron ciertos delitos que pueden ser utilizados para establecer un control indirecto de la exactitud de las respuestas dadas tanto a la encuesta de victimización como a la de delincuencia autorrevelada.

Los resultados muestran una reducción muy importante de los delitos contra la propiedad y confirman que los delitos violentos (le-

\section{Boletín Criminológico $N^{o} 39$ Febrero


Cuadro 1. Prevalencia de las victimizaciones sufridas antes de la entrada en el programa y después de un año en tratamiento, según la implicación en la delincuencia -delincuentes vs.no delincuentes-(periodo de referen-

cia: los 6 meses anteriores a la entrevista correspondiente; $n=319$ )

\begin{tabular}{|l|ccc|crr|}
\hline & \multicolumn{3}{|c|}{ Antes } & \multicolumn{3}{|c|}{ Después } \\
\hline \multicolumn{1}{|l|}{ Victimizaciones } & $\begin{array}{l}\text { no delinc. } \\
(\mathrm{n}=101)\end{array}$ & $\begin{array}{c}\text { delinc. } \\
(\mathrm{n}=217)\end{array}$ & $p$ & $\begin{array}{l}\text { no delinc. } \\
(\mathrm{n}=223)\end{array}$ & $\begin{array}{c}\text { delinc. } \\
(\mathrm{n}=96)\end{array}$ & $p$ \\
Robo con violencia o intimidación & 6.0 & 13.4 & .05 & 2.7 & 10.9 & $<.01$ \\
Lesiones corporales graves & 3.0 & 3.7 & N.S. & 1.8 & 4.3 & N.S. \\
Agresiones sexuales & 0.0 & 1.9 & N.S. & 0.0 & 4.4 & $<.01$ \\
Estafa en la compra de drogas & 46.0 & 59.7 & $<.05$ & 12.6 & 31.5 & $<.01$ \\
Robo de dinero u objetos & 12.0 & 28.7 & $<.01$ & 8.6 & 22.8 & $<.01$ \\
\hline
\end{tabular}

siones corporales y agresiones sexuales) no parecen tener una relación directa con el estilo de vida de los toxicómanos puesto que el porcentaje de víctimas de dichos delitos es extremadamente bajo (con un máximo de 11 y 4 personas respectivamente). La importancia del estilo de vida es puesta de relieve en el cuadro 1 que permite observar la relación entre delincuencia y victimización. En este cuadro los participantes han sido divididos en dos grupos: el primero de ellos ("no delincuentes") contiene aquellos que manifiestan no haber cometido ninguna infracción en el período de tiempo estudiado; el segundo ("delincuentes") contiene los que, por el contrario, admiten haber cometido una infracción en dicho período.

Tanto en el período anterior a la entrada en el programa como un año más tarde, el porcentaje de personas víctimas de delitos es mayor entre aquellos participantes que admiten haber cometido infracciones que entre aquellos que afirman lo contrario. Este resultado confirma la importancia del estilo de vida como factor explicativo de las victimizaciones (obsérvese que en la mayoría de los ca- sos la diferencia no es significativa para los delitos ajenos a dicho estilo). Por otro lado, la influencia del programa se hace sentir en los dos grupos, puesto que ambos han registrado una disminución de las victimizaciones, lo que sugiere un verdadero cambio en el comportamiento de los participantes, quienes parecen haberse alejado del ambiente marginal que rodea a la toxicomanía.

\section{Conclusión}

Todos los indicadores de la delincuencia utilizados en esta investigación registran una disminución significativa de los delitos cometidos por las personas en tratamiento. Los contactos con la policía se redujeron en un $60 \%$ y las condenas penales en un $80 \%$. Las encuestas de delincuencia autorrevelada muestran una disminución aún más importante de las infracciones cometidas, principalmente de los delitos graves contra la propiedad y del tráfico de drogas.

Por su parte, las encuestas de victimización reflejan un cambio muy importante en el estilo de vida de los participantes, quienes constituían un grupo extremadamente marginal de la población antes de

La revista Derecho Penal y Criminología invita a los lectores de este boletín a hacerle llegar sus estudios sobre temas de interés criminológico. Los manuscritos deben presentar resultados de proyectos empíricos interpretados en un marco teórico, y tener una extensión entre 15 y 30 páginas. Las preguntas y sugerencias pueden dirigirse a Mariano Melendo (91 6406020; e-mail: mmelendo@ sr.uned.es) o a Per Stangeland (952 132333; email: stangeland@uma.es). entrar en el programa. Este resultado es confirmado por los estudios realizados por otros Institutos que muestran también una neta mejoría de la situación socioeconómica y de la salud de los pacientes. Señalemos además que el porcentaje de personas que abandonaron el tratamiento con heroína es relativamente bajo, lo que de por sí constituye un éxito dado que uno de los principales problemas que presenta esta categoría de toxicómanos es la falta de motivación para continuar en tratamiento.

Los resultados presentados nos permiten afirmar que el tratamiento con heroína constituye una alternativa eficaz para las personas implicadas gravemente en la toxicomanía y la delincuencia y para quienes los tratamientos convencionales han fracasado. A largo plazo, las mejorías observadas en los distintos indicadores médicos, económicos y sociales pueden favorecer en estas personas la decisión de abandonar definitivamente la toxicomanía y la marginalidad que la acompaña. Al mismo tiempo, la reducción de los delitos cometidos permite mejorar la calidad de vida del resto de la población en las ciudades gravemente afectadas por el problema de la toxicomanía. Desde este punto de vista, el programa de prescripción médica de heroína aplicado en Suiza es sin duda una de las medidas más eficaces intentadas en el terreno de la prevención de la delincuencia.

\section{Boletín

